\title{
Modeling of Transient Thermal Conditions in Cutting
}

\author{
T. Augspurger ${ }^{1}$, F. Klocke ${ }^{1}$, B. Döbbeler ${ }^{1}$, M. Brockmann ${ }^{1}$, S. Gierlings ${ }^{2}$ and A. Lima ${ }^{1}$ \\ 1. Laboratory for Machine Tools and Production Engineering, RWTH Aachen University, Aachen 52056, Germany \\ 2. Fraunhofer Institute for Production Technology IPT, RWTH Aachen University, Aachen 52074, Germany
}

\begin{abstract}
The thermal conditions like the temperature distribution and the heat fluxes during metal cutting have a major influence on the machinability, the tool lifetime, the metallurgical structure and thus the functionality of the work piece. This in particular applies for manufacturing processes like milling, drilling and turning for high-value turbomachinery components like impellers, combustion engines and compressors of the aerospace and automotive industry as well as energy generation, which play a major role in modern societies. However, numerous analytical and experimental efforts have been conducted in order to understand the thermal conditions in metal cutting, yet many questions still prevail. Most models are based on a stationary point of view and do not include time dependent effects like in intensity and distribution varying heat sources, varying engagement conditions and progressive tool wear. In order to cover such transient physics an analytical approach based on Green's functions for the solution of the partial differential equations of unsteady heat conduction in solids is used to model entire transient temperature fields. The validation of the model is carried out in orthogonal cutting experiments not only punctually but also for entire temperature fields. For these experiments an integrated measurement of prevailing cutting force and temperature fields in the tool and the chip by means of high-speed thermography were applied. The thermal images were analyzed with regard to thermodynamic energy balancing in order to derive the heat partition between tool, chips and workpiece. The thus calculated heat flow into the tool was subsequently used in order to analytically model the transient volumetric temperature fields in the tool. The described methodology enables the modeling of the transient thermal state in the cutting zone and particular in the tool, which is directly linked to phenomena like tool wear and workpiece surface modifications.
\end{abstract}

Key words: Metal cutting, infrared thermography, heat sources, transient temperature fields, model based on Green's functions.

\section{Introduction}

The temperature distribution and related heat flows in the cutting zone are of significant importance for an economical design of cutting processes regarding tool lifetime, machining time and work piece quality [1-7]. Many tool wear phenomena during cutting are directly or indirectly temperature driven. Thus the applied tool material, coating and parameter setting have to be tuned with respect to the expected thermal conditions. Eventually the thermal loads on the work piece summarized under the concept of process signatures may affect its functional performance in form of alterations in the material structure like residual stresses, hardness and embrittlement [8].

This paper focuses on the thermal conditions in the tool, affiliated heat sources, resulting heat flows and

Corresponding author: Thorsten Augspurger, Dipl. Wirt. Ing., research fields: cutting processes, temperatures, heat partitions. temperature fields. Especially the time regime will be taken into consideration as the temporal development of temperature fields in the tool that are focused, which are calculated by a model based on Green's functions. As inputting for the model spatial distributed planar heat sources are applied on a semi-infinite corner (as an idealized cutting edge). Though many models exist in order to calculate temperature fields resulting from heat sources, the form, distribution and intensity of heat sources in the primary and secondary shear zone which may describe the observed temperature distributions are not explored to a satisfactory extend $[9,10]$. The paper is organized as follows: Section 2 outlines the state of the art regarding analytical modeling of temperature fields and heat partitions as well as the research regarding the spatial distribution of heat sources on the rake face. Section 3 describes the conducted experiments as well as the methods for data analysis. Section 4 shows a representative extraction of the derived results. 


\section{State of the Art}

\subsection{Modeling Transient Temperature Fields in Metal Cutting with Green's Functions}

Numerous analytical and experimental efforts have been done in order to understand the thermal conditions in metal cutting. Most of these models are based on the Jaeger solution [11] for moving heat sources, which gives a solution for the stationary temperature field caused by a moving heat source on a semi-infinite solid. This approach can be used to calculate temperature fields in mathematical adequately linked sub regions of the cutting zone like the tool, the workpiece and the chip region considering boundary conditions (e.g., adiabatic, Neumann). In 1999 Komanduri and Hou [10, $12,13]$ proposed a model based on Hahn's work [14], which enables the calculation of temperature fields in the entire cutting zone for the stationary state, in two dimensions, under the assumption of idealized engagement conditions and heat distributions. Most of the developed temperature models are not able to directly link process parameters to thermal conditions, but only idealized heat sources with given intensity and distribution. In their work of 1947 Carslaw and Jaeger [11] suggested the possibility to use Green's functions in order to solve inhomogeneous transient heat conduction problems for the applications in one-, twoand three-dimensional problems with heat generation. In his work Özisik [15] gives a good overview of the method. In 1992 Stephenson [16] picked up the approach in order to model the punctual transient temperature in the tool during interrupted cutting. In his experiments he investigated the influence of uniform, linear and exponential heat source distributions along the rake face as well as time varying heat source intensities in form of square wave heat inputs. Regarding to Stephenson [16] the Green's function for the temperatures in a semi-infinite corner due to an instantaneous point source at time $t$ at the surface point $\mathrm{x}=\mathrm{x}_{\mathrm{p}}, \mathrm{y}=\mathrm{y}_{\mathrm{p}}, \mathrm{z}=0$, assuming adiabatic conditions at the boundaries can be obtained by multiplying the Green's function for three mutually perpendicular instantaneous plane sources in semi-infinite half spaces which intersect to form an eighth space or corner (Fig. 1). Thus the temperature field $\mathrm{T}(\mathrm{x}, \mathrm{y}, \mathrm{z}, \mathrm{t})$ in the corner as a solution of

$$
\begin{gathered}
\frac{\partial^{2} \mathrm{~T}}{\partial \mathrm{x}^{2}}+\frac{\partial^{2} \mathrm{~T}}{\partial \mathrm{y}^{2}}+\frac{\partial^{2} \mathrm{~T}}{\partial \mathrm{z}^{2}}=\frac{1}{\alpha} \cdot \frac{\partial \mathrm{T}}{\partial \mathrm{t}} \\
-\mathrm{k} \cdot \frac{\partial \mathrm{T}}{\partial \mathrm{x}}=\dot{\mathrm{Q}}(\mathrm{x}, \mathrm{y}, \mathrm{t}) ; \mathrm{z}=0 ; 0 \leq \mathrm{y} \leq \mathrm{L}_{\mathrm{y}}
\end{gathered}
$$

can be calculated by

$$
\begin{aligned}
& T(x, y, z, t) \\
& =\frac{\alpha}{k} \int_{0}^{t} \int_{0}^{L_{x}} \int_{0}^{L_{y}} \Theta_{G}\left(x, y, z, t, x_{p}, y_{p}, 0, D\right) \\
& \cdot Q\left(x_{p}, y_{p}, \tau\right) d y_{p} d x_{p} d \tau
\end{aligned}
$$

with

$$
\begin{aligned}
& \Theta_{G}\left(x, y, z, t, x_{p}, y_{p}, 0, D\right) \\
& =\frac{2}{(\sqrt{\pi} \cdot D)^{3}} \cdot \exp \left(\frac{-z^{2}}{D^{2}}\right) \\
& \cdot\left[\exp \left\{-\frac{\left(x+x_{p}\right)^{2}}{D^{2}}\right\}+\exp \left\{-\frac{\left(x-x_{p}\right)^{2}}{D^{2}}\right\}\right] \\
& \cdot\left[\exp \left\{-\frac{\left(y+y_{p}\right)^{2}}{D^{2}}\right\}+\exp \left\{-\frac{\left(y-y_{p}\right)^{2}}{D^{2}}\right\}\right]
\end{aligned}
$$

as the Green's function and

$$
\mathrm{D}=2 \cdot \sqrt{\alpha(\mathrm{t}-\tau)}
$$

with Q $\left(\mathrm{x}_{\mathrm{p}}, \mathrm{y}_{\mathrm{p}}, \mathrm{t}\right)$ and $L_{x}, L_{y}$ as the spatial and temporal distribution of the heat source, which is of particular significance for the resulting temperature field, especially for the maximum temperature generated. Stephenson obtained a good validation of the model for

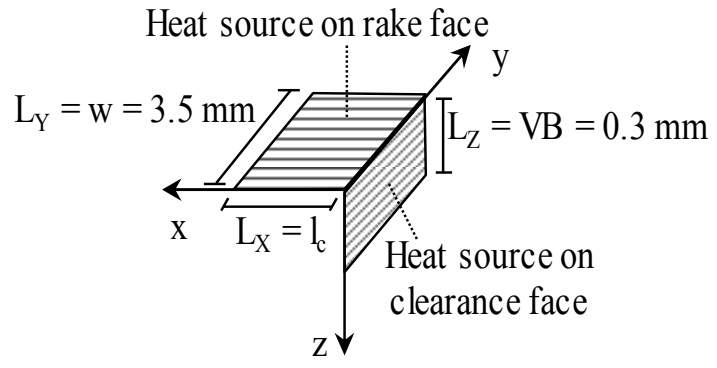

Fig. 1 Semi-infinite corner. 
the punctual case. So did Refs. [17-19], who used the proposed model in order to predict punctual temperatures in the milling tool, which have been either measured by integrated thermocouples or pyrometry. Jiang et al. [17] and Sato et al. [18] integrated an analytical model for the contact length in order to perform a time dependent spatial heat source distribution due to the varying uncut chip thickness in milling. The heat flux $q_{c}$ per unit area on the rake face was determined by force measurements and assumed as uniformly distributed. No heat partition ratio was considered, so the total process power was assumed to be conducted into the tool. Only one heat source on the rake face was applied which is not sensible due to existing frictional heat source on the clearance face. For not an entire temperature field was observed, but only punctual temperatures, the demand for an additional heat source on the clearance face was not revealed.

\subsection{Spatial Distribution of Heat Sources on Tool-Chip Interface (Rake Face)}

The temperature rise in metal cutting is caused by three principal heat sources, the shear plane heat source at the primary shear plane and the frictional heat sources at the tool-chip interface and on the clearance face. All of them are ideally considered as two dimensional.

The heat flux distribution, the heat partition and the temperature rise distribution at the tool-chip interface is a subject of research since temperatures in cutting have been investigated [12]. In 1937 Blok [20] explored the temperature rise in the interface of two bodies in relative motion and invented the principle of heat partition. Later in 1951 Chao and Trigger [21] applied his principle to the cutting process in order to model the temperature rise at the tool rake face due to frictional contact in addition to the temperature rise due to chip deformation at the shear zone. They considered the frictional heat source between chip and the tool as a heat source that is moving in relation to the chip and at the same time stationary in relation to the tool. They thus calculated the average heat partition fractions for the chip and the tool and in a next step the average temperature at the tool-chip interface. In their further work they found out that the heat flux distribution at the contact is rather non-uniform than uniform [22].

In his publication of 1963 Boothroyd [23] proposed a numerical method in order to draw conclusions for the heat source distribution in the friction zone. $\mathrm{He}$ assumed that the actual heat generation rate in the region of the tool rake face may be a combination of a distributed heat source due to chip deformation and a plane heat source due to sliding friction. He further examined the influence of a triangular and a rectangular uniform distribution of the heat source and came to the conclusion that a triangular deformation zone with the maximum at the tool tip gives a reasonable approximation for his given case (orthogonal cutting of mild steel, preheated). The research community increasingly distanced themselves from the idea of planar heat sources, so did Refs. [10, 12, 13]. According to the current state of the art, besides the primary shear zone, a secondary shear zone at the tool-chip interface and further plastic deformation with volumetric heat generation is supposed [24]. The shape of this zone is directly connected to the material properties, the conditions at the interface and the occurrence of sliding respectively sticking friction, which depends of the parameter setting and the material pairing [25].

\section{Experiments, Post Processing and Modelling}

\subsection{Experimental Setup}

The orthogonal cutting tests were carried out on AISI 1045 (C45E normalized, width of cut $\mathrm{w}=3.5 \mathrm{~mm}$ ) with cutting speeds $v_{c}=100 \mathrm{~m} / \mathrm{min}, 150 \mathrm{~m} / \mathrm{min}$ and uncut chip thickness $\mathrm{h}_{\mathrm{sp}}=0.2 \mathrm{~mm}, 0.3 \mathrm{~mm}$. As tool an uncoated carbide insert (Sandvik H13A) with a rake angle $\gamma_{n}=6^{\circ}$, a clearance angle $\alpha_{n}=3^{\circ}$, a cutting edge rounding of $\mathrm{r}_{\beta} \leq 5 \mu \mathrm{m}$ and a width of $4.4 \mathrm{~mm}$ was used. 
The cutting took place a distance of $0.1 \mathrm{~mm}$ from the outer rim of the tool. The cutting trails were conducted without coolant or lubrication on a broaching machine, which served through a customized planning configuration as a fundamental test bench. The work piece of the dimensions $200 \times 3.5 \times 80 \mathrm{~mm}^{3}$ was moved in a linear motion. A cut of $200 \mathrm{~mm}$ length was made in one direction by the tool, which was lowered before each cutting stroke. The broaching machine not only provided high positioning accuracy and rigidity but also a very good accessibility for the thermal camera. The high speed infrared camera of type FLIR SC7600 (framerate $328 \mathrm{fps}$ at $640 \times 512$ pixels) recorded the infrared radiation of the fixed tool during the process. The surface of the work piece and tool were coloured with a black coating whose emissivity coefficient was determined in previous investigations as approximately 0.85 for the relevant temperature range. A preceding Ref. [26], which focused on the measurement of temperature fields and heat partition ratios in the cutting zone, was also based on this experiment.

\subsection{Utilization of Green's Functions for Modelling Transient Temperature Fields in Orthogonal Cutting}

In this investigation the research question is raised, if entire temperature fields in orthogonal cutting can be modelled with Green's functions close to reality in the time domain. It is further questioned which spatial distribution of heat sources at the cutting edge can be deduced by these fields. So an inverse problem is addressed, which does not have a unique mathematical solution. Potential heat sources as the causes of the temperature field are calculated by simple iteration. The proposed approach uses Eq. (2) in order to calculate a volumetric temperature distribution in the semi-infinite corner while the extensions of the heat sources $L_{x}, L_{y}, L_{z}$ are chosen with respect to the measured contact length $l_{c}$ for the process parameter setting and the width of cut $\mathrm{w}=3.5 \mathrm{~mm}$. For the intensity of the applied heat sources at the rake face exponential distributions were chosen. In addition, a secondary heat source on the clearance face was introduced, whose intensity was uniformly distributed over an estimated area of $0.3 \times 3.5 \mathrm{~mm}^{2}$ and estimated to $40 \mathrm{MW} / \mathrm{m}^{2}$. This secondary heat source can be super positioned with the first one in the model due to the linear and homogeneous governing equations [16]. The total intensity of the heat sources is measured by the post processing method proposed in Ref. [26]. This method calculates the heat flows and heat partition among tool, chip and work piece in the cutting zone by a heat balance based on thermographic images, which are subjected to a subsequent regression, in order to smooth irregularities. The heat flow into the tool during cutting is then calculated by

$$
\begin{aligned}
\dot{\mathrm{Q}}_{\text {tool,in }} & =\frac{\mathrm{dU}}{\mathrm{dt}}+\dot{\mathrm{Q}}_{\text {tool,out }} \\
\dot{\mathrm{Q}}_{\text {tool,out }} & =\lambda_{\text {tool }} \cdot \mathrm{l}_{\text {iso }} \cdot \frac{\mathrm{dT}}{\mathrm{dn}} \\
\frac{\mathrm{dU}}{\mathrm{dt}} & =\mathrm{c}_{\mathrm{p}}(\mathrm{T}) \cdot \frac{\mathrm{dT}}{\mathrm{dt}}
\end{aligned}
$$

This heat flow varies over time and reaches a stationary state in the given case [26]. The thus calculated heat flow in the stationary state, disengaged from time, is used as input for the model. So the heat sources as input for the model are prone to a spatial and not temporal distribution, which would be closer to reality but more complex. In this way the model calculates a transient volumetric temperature distribution. The computed temperature fields in the $\mathrm{x}-\mathrm{z}$ plane were compared qualitatively and quantitatively to the measured fields at the tool side with regard to maximum temperatures at the tool tip, direction of isotherms and gradients. Regarding the physical tool material properties mean values for thermal diffusivity $\mathrm{a}=2 \times 10^{-5} \mathrm{~m}^{2} / \mathrm{s}$ and heat conductivity $75 \mathrm{~W} / \mathrm{mK}$ for the relevant temperature range were chosen according to Ref. [27], which experimentally determined the affiliated series of values for the same tool material. Due to the assumption of an idealized corner in the model a volumetric deviation of approx. $6 \%$ occurs, which certainly restricts the comparability. 


$$
\mathrm{h}_{\mathrm{sp}}=200 \mu \mathrm{m} \text { and } \mathrm{v}_{\mathrm{c}}=\mathbf{1 5 0} \mathrm{m} / \mathrm{min}
$$

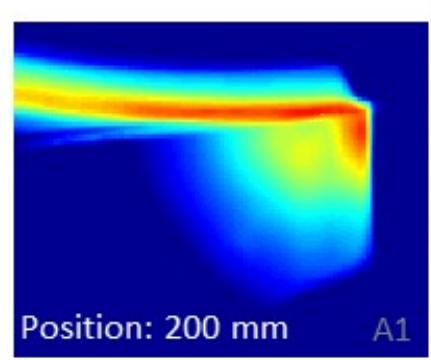

$\mathrm{x}[\mathrm{mm}]$
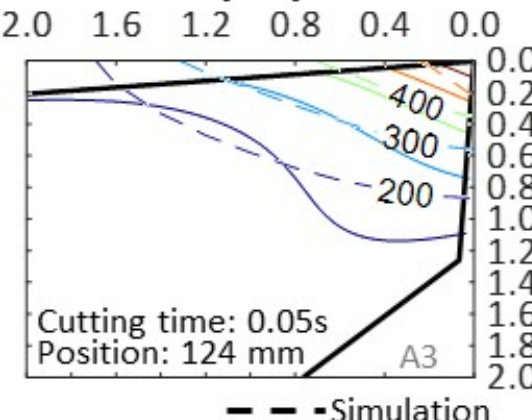

- - -Simulation $\mathrm{x}[\mathrm{mm}]$

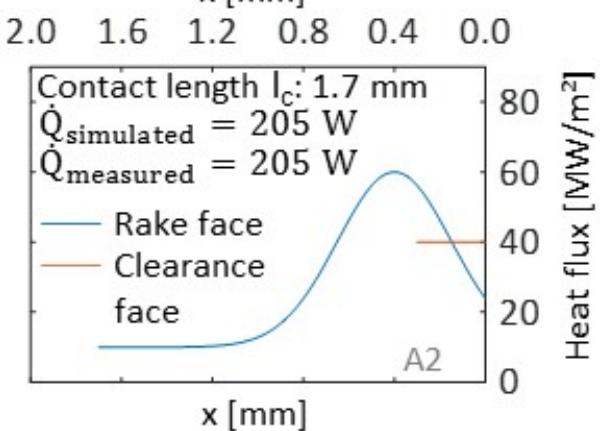

$\begin{array}{llllll}2.0 & 1.6 & 1.2 & 0.8 & 0.4 & 0.0\end{array}$

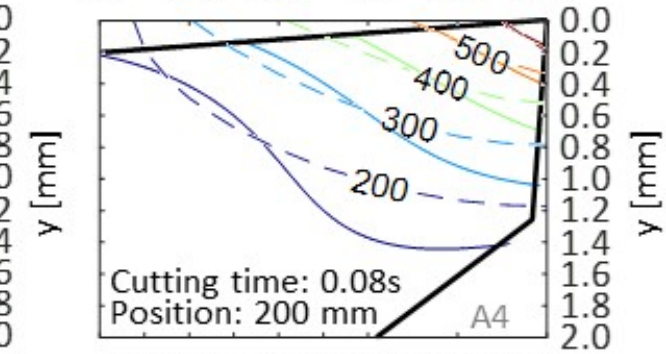

Regression of measurement

and $v_{c}=100 \mathrm{~m} / \mathrm{min}$

$\left[{ }^{\circ} \mathrm{C}\right]$

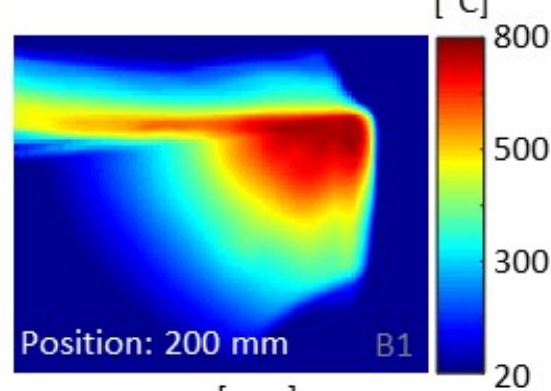

$\mathrm{x}[\mathrm{mm}]$

$$
\begin{array}{llllll}
2.0 & 1.6 & 1.2 & 0.8 & 0.4 & 0.0
\end{array}
$$

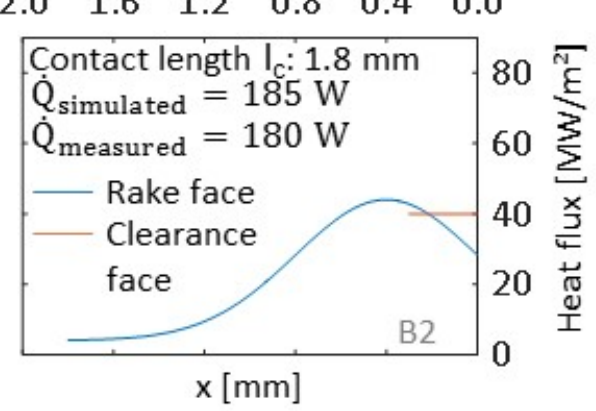

$\begin{array}{llllll}2.0 & 1.6 & 1.2 & 0.8 & 0.4 & 0.0\end{array}$

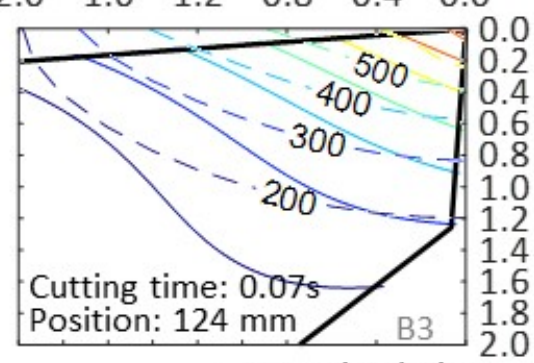

- - -Simulation $\begin{array}{llllll}2.0 & 1.6 & 1.2 & 0.8 & 0.4 & 0.0\end{array}$

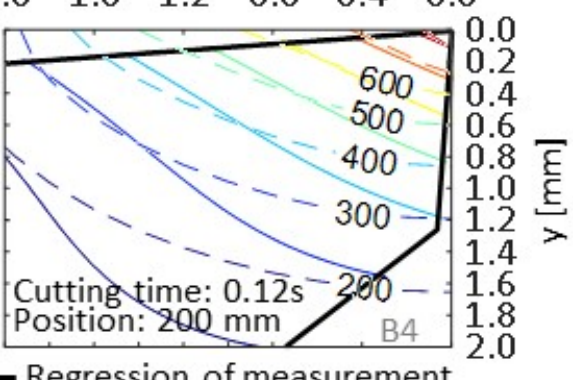

Fig. 2 (A) Images for $h_{s p}=200 \mu \mathrm{m}$ and $v_{c}=150 \mathrm{~m} / \mathrm{min}$, (B) images for $h_{\text {sp }}=300 \mu \mathrm{m}$ and $v_{c}=100 \mathrm{~m} / \mathrm{min}$, (1) combined thermal image for $200 \mathrm{~mm}$ position, (2) heat source distribution along the rake face and clearance face, (3) temperature distribution for $124 \mathrm{~mm}$ position (regression of measurement and simulation), (4) temperature distribution for $200 \mathrm{~mm}$ position (regression of measurement and simulation). 


\section{Results}

Fig. 2 shows the measured and simulated transient temperature fields for two different $v_{c}$ and $h_{s p}$ at two cutting times. It further shows the infrared picture and the heat source distribution applied on the rake face for the model. The infrared pictures show higher temperatures in the area of the tool tip compared to Ref. [26] due to the local $\left(0.5 \times 0.5 \mathrm{~mm}^{2}\right)$ assumption of an emissivity of 0.5 , which is sensible due to observed chipping of coating at the tool tip. Thus no original infrared picture is shown but the synthesis of two considering two different emissivities.

The main results achieved in this investigation can be summarized as follows:

- The model based on Green's function can be used to predict transient temperature fields close to reality for orthogonal cutting, qualitatively and quantitatively. Thus it can be potentially used to predict entire temperature fields in interrupted cutting processes, like milling. The prediction of the time dependence and spatially distribution of the projected affiliated heat sources and, in particular, the heat partition ratios in the cutting zone connected to the parameter setting of the process will be significant.

- The shown possible solutions of the inverse problem lead to exponentially distributed projected heat sources at the tool-chip-contact area (in reality it is to be assumed to be rather volumetric than planar) and simultaneously to a uniformly distributed heat source on the clearance face. The potential solutions of the inverse problem remain uncertain. In the investigations it was not possible to model realistic temperature fields with a sole heat source at the rake face.

- The shapes of the isotherms deviate between the measurement and the model. This can be either explained by the subsequent regression of the measured field, which leads to a distortion of the isotherms or the differences in the shape and volume of the real cutting edge and the semi-infinite corner. As assumed before in Ref. [26] the measured temperature at the tool tip in Ref. [26] has been underestimated, due to a wrong assumption regarding the emissivity. This insignificantly changes the results of the energy balance, but explains deviations regarding the depicted thermographic pictures.

\section{Acknowledgments}

The authors wish to thank the German Research Foundation (DFG) for funding the transregional Collaborative Research Center SFB/TR 96 "Thermo-Energetische Gestaltung von Werkzeugmaschinen" (Aachen, Chemnitz, Dresden), subproject A02.

\section{References}

[1] Nakayama, K., Shaw, M. C., and Brewer, R. C. 1966. "Relationship between Cutting Forces, Temperature, Built-up Edge and Surface Finish." Annals of the CIRP 14: 211-23.

[2] Shouckry, A. S. 1982. "The Effect of Cutting Conditions on Dimensional Accuracy." Wear 80 (2): 197-205.

[3] Trent, E. M. 1988. "Metal Cutting and the Tribology of Seizure: I. Seizure in Metal Cutting." Wear 128 (1): 29-45.

[4] Trent, E. M. 1988. "Metal Cutting and the Tribology of Seizure: II. Movement of Work Material over the Tool in Metal Cutting." Wear 128 (1): 47-64.

[5] Trent, E. M. 1988. "Metal Cutting and the Tribology of Seizure: III. Temperatures in Metal Cutting." Wear 128 (1): 65-81.

[6] Taylor, F. W. 1907. "On the Art of Cutting Metals." Trans. ASME 28: 32-248.

[7] Klocke, F., Brockmann, M., Gierlings, S., and Veselovac, D. 2014. "Force-Based Temperature Modelling for Surface Integrity Prediction in Broaching Nickel-Based Alloys." Procedia CIRP 13: 314-9.

[8] Brinksmeier, E., and Klocke, F. 2014. "Process Signatures." Procedia CIRP 13: 429-34.

[9] Da Silva, M. B., and Wallbank, J. 1999. "Cutting Temperature: Prediction and Measurement Methods-A Review." Journal of Materials Processing Technology 88 (1-3): 195-202.

[10] Komanduri, R., and Hou, Z. B. 2000. "Thermal Modeling of the Metal Cutting Process: Part I-Temperature Rise Distribution due to Shear Plane Heat Source." Int. J. Mech. Sci. 42 (9): 1715-52.

[11] Jaeger, J. C. 1942. "Moving Sources of Heat and the Temperatures at Sliding Contacts." Proceedings of Royal 
Society of NSW 76: 203-24.

[12] Komanduri, R., and Hou, Z. B. 2001. "Thermal Modeling of the Metal Cutting Process-Part II: Temperature Rise Distribution due to Frictional Heat Source at the Tool-Chip Interface.” Int. J. Mech. Sci. 43 (1).

[13] Komanduri, R., and Hou, Z. B. 2001. "Thermal Modeling of the Metal Cutting Process-Part III: Temperature Rise Distribution due to the Combined Effects of Shear Plane Heat Source and the Tool-Chip Interface Frictional Heat Source.” Int. J. Mech. Sci. 43 (1): 89-107.

[14] Hahn, R. S. 1951. "On the Temperature Development at the Shear Plane in Metal Cutting Processes." In Proceedings of the 1st US National Congress of Appl. Mech (1951), 661-6.

[15] Özisik, M. N. 1993. Heat Conduction. New York: Wiley, 214-56.

[16] Stephenson, D. A. 1992. "Tool Temperatures in Interrupted Metal Cutting." Journal of Engineering for Industry 114 (2): 127-36.

[17] JIANG, F. L., LIU, Z. Q., WAN, Y., and SHI, Z. Y. 2013. "Analytical Modelling and Experimental Investigation of Tool and Workpiece Temperatures for Interrupted Cutting 1045 Steel by Inverse Heat Conduction Method." Journal Materials Processing Technology 6: 887-94.

[18] Sato, M., Tamura, N., and Tanaka, H. 2011. "Temperature Variation in the Cutting Tool in End Milling." Journal of Manufacturing Science and Engineering 133 (2).

[19] Karaguzel, U., and Bakkal, M. 2016. "Modeling and
Measurement of Cutting Temperatures in Milling." Procedia CIRP 46: 173-6.

[20] Blok, H. 1938. "Theoretical Study of Temperature Rise at Surfaces of Actual Contact under Oiliness Lubricating Conditions." In Proceedings of the General Discussion on Lubrication and Lubricants, London, 22-235.

[21] CHAO, B. T., and Trigger, K. J. 1951. "An Analytical Evaluation of Metal Cutting Temperature.” Trans. ASME 73: 57-68.

[22] Chao, B. T., and Trigger, K. J. 1955. "Temperature Distribution at the Tool-Chip Interface in Metal Cutting." Trans. ASME 77: 1107-21.

[23] Boothroyd, G. 1963. "Temperatures in Orthogonal Metal Cutting." Pro Institution of the Mechanical Engineers.

[24] Klocke, F., and König, W. 2008. Fertigungsverfahren-Drehen, Fräsen, Bohren. Springer 8. Auflage, 50-3.

[25] Abouridouane, M., Klocke, F., Lung, D., and Veselovac, D. 2015. "The Mechanics of Cutting: In-situ Measurement and Modeling." Procedia CIRP 31: 246-51.

[26] Klocke, F., Döbbeler, B., and Augspurger, T. 2016. "Experimental Investigation of Temperatures and Heat Flows for Orthogonal Cutting 1045 Steel by Thermal Imaging." HSM.

[27] Puls, H., Klocke, F., and Veselovac, D. 2015. "FEM-Based Prediction of Heat Partition in Dry Metal Cutting of AISI 1045." The International Journal of Advanced Manufacturing Technology 86 (1): 737-45. 